\title{
The Thermal Balance of Venus in Light of the Pioneer Venus Mission
}

\author{
M. G. Tomasko, ${ }^{1}$ Peter H. Smith, ${ }^{1}$ V. E. Suomi, ${ }^{2}$ L. A. Sromovsky, ${ }^{2}$ H. E. ReVercomb, ${ }^{2}$ \\ F. W. Taylor, ${ }^{3}$ D. J. Martonchik, ${ }^{4}$ A. Seiff, ${ }^{5}$ R. Boese, ${ }^{5}$ J. B. Pollack, \\ A. P. INGERSOLl, ${ }^{6}$ G. SCHUBERT, ${ }^{7}$ AND C. C. COVEY $^{7}$
}

\begin{abstract}
Instruments flown on the Pioneer Venus orbiter and probes measured many of the properties of the atmosphere of Venus which control its thermal balance and support its high surface temperature. Estimates based on orbiter measurements place the effective radiating temperature of Venus at $228 \pm 5 \mathrm{~K}$, corresponding to an emission of $153 \pm 13 \mathrm{~W} / \mathrm{m}^{2}$, and the bolometric Bond albedo at $0.80 \pm 0.02$, corresponding to a solar energy absorption of $132 \pm 13 \mathrm{~W} / \mathrm{m}^{2}$. Uncertainties in these preliminary values are too large to interpret the flux difference as a true energy imbalance. A mode of submicron particles is suggested as an important source of thermal opacity near the cloud tops to explain the orbiter and probe thermal flux measurements. Comparison of the measured solar flux profile with thermal fluxes computed from the measured temperature structure and composition shows that the greenhouse mechanism explains essentially all of the $500 \mathrm{~K}$ difference between the surface and radiating temperatures of Venus. Precise comparison of the observed and computed value of this difference is hindered by uncertainties in the local variability of $\mathrm{H}_{2} \mathrm{O}$ and in the thermal opacity of $\mathrm{CO}_{2}$ and $\mathrm{H}_{2} \mathrm{O}$ at high temperature and pressure. The directly measured thermal flux profiles at the small probe sites are surprisingly large and variable in the lower atmosphere. Observed zonal and meridional circulation are qualitatively as required to produce the observed uniformity of temperature structure. However, the present lack of quantitative estimates of the horizontal and vertical dynamical heat transports implied by these measurements is a significant gap in the understanding of the thermal balance of the atmosphere of Venus.
\end{abstract}

\section{INTRODUCTION}

Venus has been known for many years to have a remariably high surface temperature of some $750 \mathrm{~K}$ despite its complete coverage by highly reflective clouds which cause it to absorb less energy from the sun than does the earth. This fact is all the more remarkable in view of the overall similarity of Venus and the earth in terms of size, mass and distance from the sun.

Mechanisms that might be responsible for the thermal balance of the atmosphere of Venus have attracted considerable attention ever since the discovery of the planet's unexpectedly high surface temperature. A summary of the state of understanding of the thermal balance of Venus before the Pioneer Venus mission is given by Tomasko et al. [1977]. Briefly, it was felt that radiative processes played the key role in determining the vertical temperature structure of the atmosphere with very efficient horizontal redistribution of heat by dynamical processes required to explain the apparently small differences in temperature structure observed over the planet.

In order to improve significantly the understanding of these processes, an ambitious and coordinated program of measurements of the temperature structure, solar and thermal radia-

\footnotetext{
${ }^{1}$ Lunar and Planetary Laboratory, University of Arizona, Tucson, Arizona 85721.

${ }^{2}$ Space Science and Engineering Center, University of Wisconsin, Madison, Wisconsin 53706.

${ }^{3}$ Department of Atmospheric Physics, Oxford University, Oxford, England.

${ }^{4}$ Jet Propulsion Laboratory, Pasadena, California 91103.

${ }_{5}^{5}$ Space Sciences Division, Ames Research Center, Moffett Field, California 94035.

${ }^{6}$ Division of Geological and Planetary Sciences, California Institute of Technology, Pasadena, California 91125.

${ }^{7}$ Department of Earth and Space Sciences, University of California, Los Angeles, California 90024.
}

Copyright (C) 1980 by the American Geophysical Union. tion fields, sources of thermal opacity, cloud cover, and wind field was undertaken by the Pioneer Venus (PV) mission. Remote sensing measurements of the atmosphere above the clouds having global coverage were made from the orbiter spacecraft. Entry probes were used to measure these quantities directly from the region near the cloud tops to the ground. A total of four probes were targeted to widely spaced locations in an attempt to estimate the global scale variations of the atmosphere beneath the clouds. The entry locations of these four probes are given by Colin [this issue]. Briefly, two probes landed in daylight, the 'Sounder' probe near the equator and the 'Day' probe at $\sim-30^{\circ}$ latitude, and two on the night side of the planet, the 'Night' probe also at $\sim-30^{\circ}$ latitude and the 'North' probe at $\sim 60^{\circ}$ latitude. Thus both daynight and latitude effects could be explored in a preliminary way.

In this article we attempt to pull together the new measurements which bear on the thermal balance of Venus, and summarize our current understanding of the clarification they produce and the new questions which they raise. In the next section we review the temperature structure measurements made by PV. In section 3 we discuss the measurements relating to radiative processes - the solar and thermal flux measurements and the measurements concerning sources of thermal opacity. Section 4 discusses the new questions raised by the radiation measurements and some possible resolutions. In section 5 we briefly review the implications of the measurements concerning the dynamical state of the atmosphere for its thermal balance. A final section summarizes the important conclusions and new questions regarding the thermal balance of Venus which have resulted from the PV measurements.

\section{MEASUREMENTS OF TEMPERATURE STRUCTURE}

Extensive maps of the temperature structure above the clouds were obtained from the Pioneer Venus orbiter [Taylor et al., this issue], while radio occultation measurements pro- 


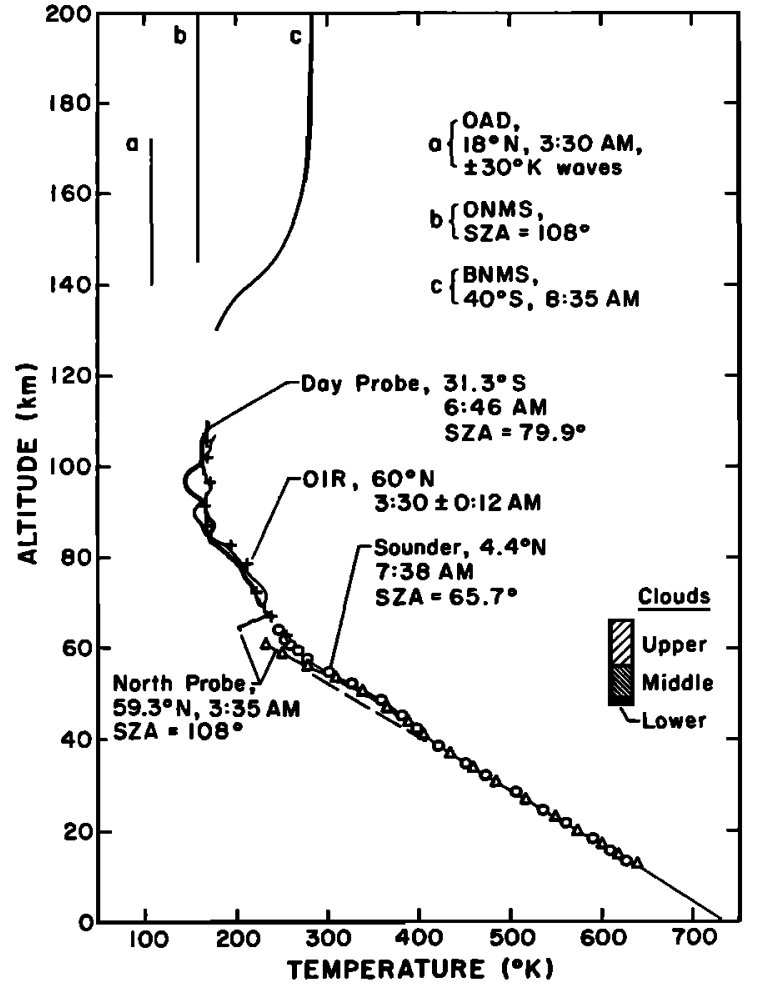

Fig. 1. Temperature profiles measured by various Pioneer Venus instruments as indicated-atmosphere drag experiment (OAD), orbiter neutral mass spectrometer (ONMS), bus neutral mass spectrometer (BNMS), orbiter infrared radiometer (OIR), and the atmospheric structure experiments on the probes.

vided limited coverage down to an altitude of about $45 \mathrm{~km}$ [Kliore and Patel, this issue]. In situ temperature measurements were obtained down to $13 \mathrm{~km}$ altitude on the four Pioneer Venus entry probes [Seiff et al., this issue; Suomi et al., 1979]. Figure 1 shows atmospheric temperature data from several Pioneer Venus experiments collected on a single graph to show profiles from 13 to $200 \mathrm{~km}$ altitude.

From the cloud levels to the surface, the mean temperature lapse rate is $\sim 7.8 \mathrm{~K} / \mathrm{km}$ on both the day and night sides of the planet at latitudes up to at least $60^{\circ}$. Above the clouds, a stratosphere extends upward to $\sim 100 \mathrm{~km}$, becoming nearly isothermal at a temperature near $170 \mathrm{~K}$. The lower boundary of this major change in lapse rate coincides with the region of sharply increased heating seen in the solar net flux profile above $56 \mathrm{~km}$ [Tomasko et al., this issue]. A prevalent feature of the cloud top region at higher latitudes is the inversion in the temperature profile at about $60 \mathrm{~km}$ shown in Figure 1 by the North probe data. This has been seen consistently in radio occultation measurements [Howard et al., 1974; Kliore and Patel, this issue], even at near equatorial latitudes, where its amplitude is smaller. It is apparently a region of adjustment in radiative balance, from the upper cloud to the haze and clear stratosphere above it. The upper atmosphere is cold relative to that of the earth, and is subject to large diurnal temperature variation [Keating et al., this issue].

The global variation in the temperature structure seen above the clouds by the orbiter infrared instrument was moderate. Temperature differences at a given altitude are no greater than a few degrees $\mathbf{K}$ from day to night at levels below $100 \mathrm{~km}$, and vary $\sim 20 \mathrm{~K}$ with latitude. The warmest regions lie over the poles [Taylor et al., 1979b, $c$, this issue]. Below the clouds, the two mid-latitude probes showed temperatures of a few degrees $\mathbf{K}$ warmer than those measured by the Sounder probe near the equator. The diurnal variation below the clouds is very small. At the North probe site, $60^{\circ}$ latitude, larger temperature differences of up to $25 \mathrm{~K}$ cooler than at lower latitudes were observed in the clouds above $55 \mathrm{~km}$ [Seiff et al., this issue]. The temperature difference measured between $60^{\circ}$ and $30^{\circ}$ latitude is approximately that needed to maintain the zonal winds in cyclostrophic balance [Seiff et al., $1979 b$, this issue], reflecting the strong coupling in the clouds between temperature structure and dynamics. Although the probe entry sites were limited to local Venus times from midnight to $8 \mathrm{~A} . \mathrm{M}$. and from $4^{\circ}$ to $60^{\circ}$ latitude, the radio occultation data extend the observations to polar latitudes. These observations show that cooling of the atmosphere in the clouds with increasing latitude continues to the poles.

Some of the most interesting results of the Pioneer Venus atmosphere structure experiments were related to atmospheric stability. Prior to Pioneer Venus, it had been thought that the deep atmosphere was generally near-adiabatic, or convective [see, e.g., Stone, 1975; Marov, 1973]. The temperature structures measured by Pioneer Venus show instead that the deep atmosphere from $\sim 26$ to $52 \mathrm{~km}$ is stable. The dashed line in Figure 1 extends the adiabatic profile upward into this stable layer. Below this deep stable layer lies a convective layer, but it only extends down to $\sim 18 \mathrm{~km}$. Underneath this is another lower-lying stable layer (at latitudes up to at least $30^{\circ}$ ). Near the surface, there is indication from Venera 9 and 10 data $[A v$ duevsky et al., 1977] of a convective layer, and in the middle cloud, there is also a thin $(\sim 2 \mathrm{~km})$ convective layer [Seiff et al., this issue]. These observations comprise an important constraint on the thermal and dynamic models, serving as a sensitive discriminant to the selection of appropriate values for the model parameters.

\section{Measurements of Radiative Processes}

\section{Global Radiative Energy Budget}

The Vortex experiment on the PV orbiter described by Taylor et al. [this issue] has mapped the thermal emission (in seven narrow passbands) and the reflected sunlight (in a broad wavelength channel) over the northern hemisphere of Venus. These data on the reflected solar and emitted thermal radiation fields at the top of the atmosphere provide the basic data needed to calculate the planetary-scale radiation budget for Venus. On the (highly probable) assumption that the sun is the only important source of energy driving the Cytherean atmospheric 'engine,' the excess of energy absorbed over that emitted in the equatorial regions must be transported to high latitudes to supply the observed deficit there. This requirement for balance is the fundamental driving force behind the general circulation on Venus, as it is on earth.

Ingoing solar radiation. The amount of solar absorption can readily be calculated using the information in Table 4 of the paper by Taylor et al. [this issue]. Sectioning the illuminated portion of the planet by meridional lines $-30^{\circ}$ and $+30^{\circ}$ longitude (solar fixed coordinate; subsolar point at $0^{\circ}$ longitude) and zonal lines equally spaced at $10^{\circ}$ intervals starting at $0^{\circ}$ latitude; the amount of absorption $\left(\mathrm{W} / \mathrm{m}^{2}\right)$ in each sector is given by

$$
F^{4}=\int_{\Omega}(E-R) d \Omega / \int_{\Omega} d \Omega
$$

where

$$
\begin{gathered}
E=\pi F \mu_{0} \\
R=\int_{0}^{2 \pi} \int_{0}^{1} I_{s}\left(\mu, \mu_{0}, \cos \Delta \phi\right) \mu d \mu d \phi
\end{gathered}
$$


$\pi F=2621 \mathrm{~W} / \mathrm{m}^{2}$, the incident solar flux at Venus, and $I_{s}$ is the intensity of sunlight scattered through an azimuth angle $\Delta \phi$. Here $\mu_{0}$ and $\mu$ are the cosines of the zenith angles of the incident and emerging beams, respectively.

Since our primary interest is in the latitudinal distribution of the ingoing and outgoing energy, the longitude bins were integrated to obtain zonal averages in each of the nine $10^{\circ}$ wide latitude bins.

Outgoing thermal radiation. The thermal component of the radiation field was obtained by making a fit of the form

$$
I(\mu)=a \mu^{b}
$$

where $a, b$ are constants, to the measured radiances $I(\mu)$. The net outward flux is then

$$
F^{\dagger}=\int_{0}^{1} I(\mu) \mu d \mu=a /(b+2)
$$

Again, an effective wavelength was selected to simplify the integration over wavelength. It was assumed that the planet radiates like a blackbody at the effective temperature of the cloud tops as measured at $11.5 \mu \mathrm{m}$; this wavelength is close to the peak of the Planck function at Cytherean bolometric temperatures. This is not as severe a simplification as it may appear at first. Although the Vortex measurements themselves demonstrate that Venus is decidedly nongray, model calculations show that the use of the brightness temperature measured at $11.5 \mu \mathrm{m}$ to calculate the total bolometric flux is accurate to within $5-10 \%$. This is to some extent due to the fact that the higher far-infrared brightness temperatures caused by the reduced opacity of the clouds at longer wavelengths, is offset fortuitously by the narrow but very opaque $15 \mu \mathrm{m} \mathrm{CO}_{2}$ band which occurs near the maximum of $I_{\lambda}$. The models referred to above are generated by retrieving the vertical temperature profile and cloud model parameters from the Vortex thermal channels. The spectrum of Venus can then be computed from such a model and integrated over wavelength to obtain the net flux. This is a convenient way of integrating the portion of the spectrum (about $30 \%$ of the total energy) actually monitored by Vortex, and estimating the rest. Since this has to be done accurately for all latitude-longitude and $\mu$ bins, this approach to a more precise calculation of the energy budget is a long-term project and will be reported in a later paper. For the present report, its use has been limited to checking the grey approximation as noted above.

The results of the radiation budget calculation are shown in

TABLE 1. Mean Zonal Radiation Budget of Venus

\begin{tabular}{cccc}
\hline $\begin{array}{c}\text { Latitude Bin, } \\
\text { degrees north }\end{array}$ & $F_{\uparrow}, \mathrm{W} / \mathrm{m}^{2}$ & $T_{e}, \mathbf{K}$ & $\boldsymbol{F}_{\downarrow}, \mathrm{W} / \mathrm{m}^{2}$ \\
\hline $0-10$ & 146.3 & 225.4 & 190.5 \\
$10-20$ & 153.4 & 228.1 & 179.7 \\
$20-30$ & 156.7 & 229.3 & 163.7 \\
$30-40$ & 158.7 & 230.0 & 126.7 \\
$40-50$ & 155.5 & 228.8 & 102.3 \\
$50-60$ & 152.0 & 227.5 & 73.0 \\
$60-70$ & 138.5 & 222.3 & 48.2 \\
$70-80$ & 143.5 & 224.3 & 36.2 \\
$80-90$ & 178.4 & 236.8 & 12.7 \\
\hline
\end{tabular}

$F_{\uparrow}$ is the total outward thermal flux, integrated over wavelength and emission angle, averaged around a latitude zone. $F_{\downarrow}$ is the absorbed solar flux in the zone. $T_{e}$ is the effective bolometric temperature of the planet for emission at all wavelengths into a hemisphere. It is not directly comparable with the brightness temperature of the planet measured at near-nadir viewing angles! The latter is typically 10-15 K warmer.

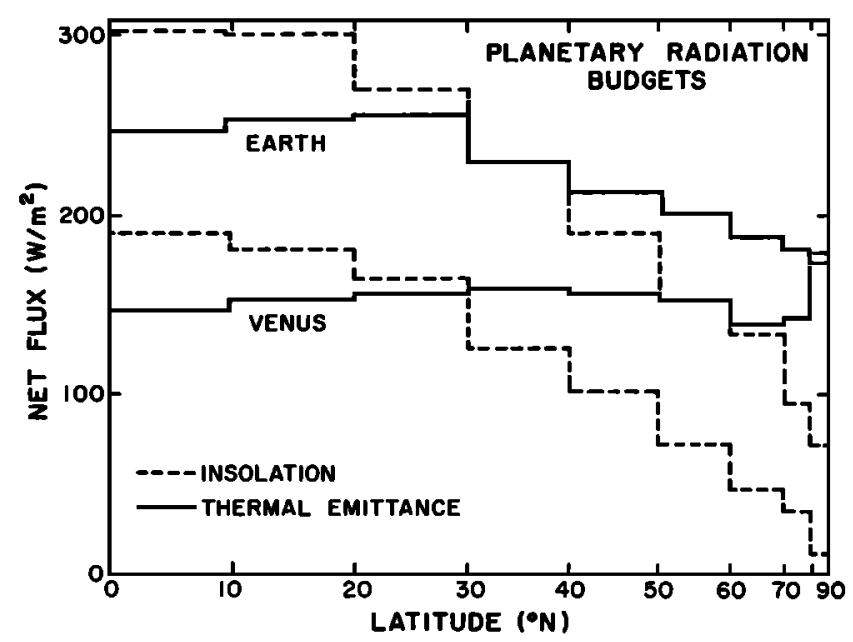

Fig. 2. Comparison of the planetary radiation budget for the earth [Vonder Haar and Suomi, 1971] and that measured by the infrared radiometer on the PV orbiter for the northern hemisphere of Venus. The longitudinally averaged thermal emission (solid line) and absorbed solar energy (dashed line) at the top of the atmosphere are shown as functions of latitude.

Table 1, and plotted in Figure 2. For comparison, Figure 2 also shows the same quantities for the earth's atmosphere, as deduced by Vonder Haar and Suomi [1971], using data from Tiros and Nimbus satellites. The total thermal flux from Venus depends significantly less strongly on latitude than does that from the earth. Other and more tentative conclusions which can be drawn from this are as follows:

1. There is an anomalously high rate of cooling at the pole on Venus, due to the relative transparency of the clouds there. However, this is nearly negligible for the energy budget of the atmosphere as a whole because its area is a small fraction of the total.

2. Both the absorbed and emitted components of the total hemispherical energy exchange, at $132 \pm 13$ and $153 \pm 13 \mathrm{~W} /$ $\mathrm{m}^{2}$, respectively (estimated errors), are only about $60 \%$ of the corresponding figures for the earth. This is contrary to a naive expectation based on the fact that the solar constant is approximately twice as great at Venus as at earth. The reason, of course, is the much larger Bond albedo of Venus. The Bond albedo is found to be $0.80 \pm 0.02$ in good agreement with $\mathbf{I r}$ vine's [1968] value of $0.77 \pm 0.07$.

3. The equivalent bolometric temperature of Venus, defined as

$$
T_{e}=\left|\frac{1}{\sigma} \int_{A v} F^{\top} d A / A \nu\right|^{1 / 4}
$$

where $\sigma$ is Stefan's constant and $A v$ is the area of the north hemisphere of Venus, is found to be equal to $228 \pm 5 \mathrm{~K}$. This value of $T_{e}$ is in approximate agreement with the value of 222 K reported by Ksanfomaliti [1980] from Venera orbiter measurements.

4. The difference between the ingoing and outgoing components for the north hemisphere corresponds to a net outflux of $20 \pm 20 \mathrm{~W} / \mathrm{m}^{2}$, or $13( \pm 13) \%$ of the power input by the sun. However, in view of the large uncertainties this cannot be interpreted as a definite energy imbalance.

\section{Vertical Profiles of the Net Radiative Flux}

Solar net flux profiles. The solar flux radiometer (LSFR) experiment aboard the Sounder probe measured the intensity 


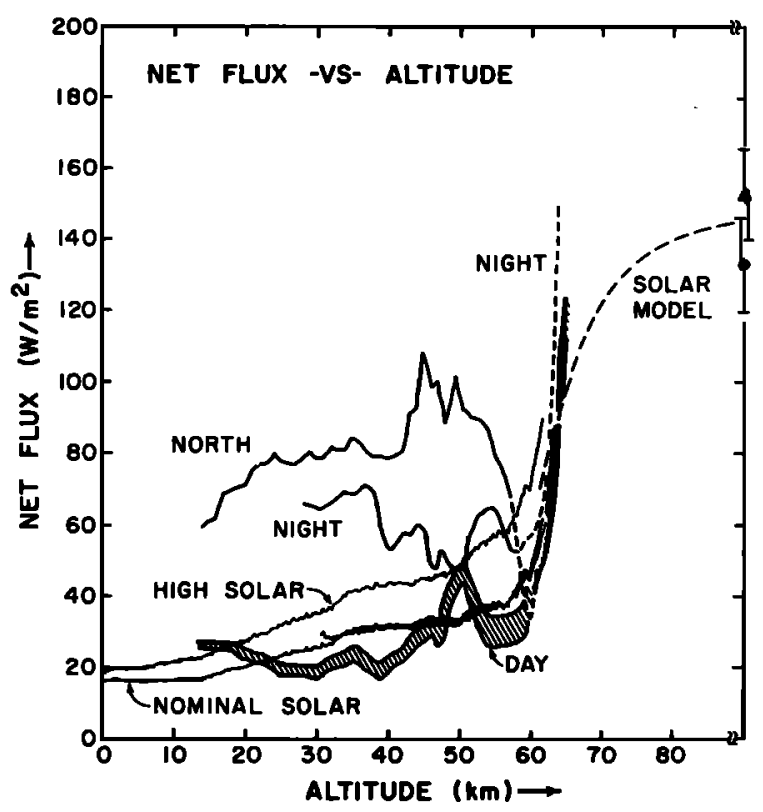

Fig. 3. The SNFR thermal net flux measurements at the Night, North, and Day probe sites are compared with the globally averaged bolometric net solar fluxes as a function of altitude. The 'broad-band' and 'combined' channels (above $35 \mathrm{~km}$ altitude) are each scaled up to include energy outside the LSFR filters and averaged over the planet using a forward scattering cloud model are shown in the curves (nearly superimposed) labeled 'nominal solar.' Also shown is the highest solar net flux profile (labeled 'high solar') consistent with relative and absolute calibration uncertainties in the LSFR upward and downward looking detectors. The dashed curve above $60 \mathrm{~km}$ altitude is a solar model calculation. The triangle corresponds to the net thermal flux and the dot to the net solar flux measured for the top of the atmosphere by the orbiter. The magnitudes of the net fluxes are plotted; the solar net flux is directed downward, and the thermal net fluxes are directed upward.

of sunlight in several directions to the vertical in a spectral band from about 0.4 to $1.0 \mu \mathrm{m}$ from $65 \mathrm{~km}$ altitude to the ground [Tomasko et al., this issue]. Between $65 \mathrm{~km}$ and about $35 \mathrm{~km}$ altitude, measurements were also made in a spectral channel from 1-1.8 $\mu \mathrm{m}$. These data have been reduced to give the upward, downward and net flux profiles at the Sounder entry site in passbands from $0.4-1.8$ and $0.4-1.0 \mu \mathrm{m}$. The absolute accuracy of these measurements is thought to be better than. $10 \%$, with relative uncertainty in the upward and downward fluxes being less than $\pm 2 \%$. Maximum errors of $2 \%$ with opposite sign in the upward and downward fluxes could cause $30 \%$ errors in the net flux at the highest altitudes where the upward flux is only some $12 \%$ less than the downward flux. At lower altitudes, the percentage difference between the upward and downward fluxes increases until the downward flux is about a factor 6 greater than the upward flux at the ground and relative calibration uncertainties are insignificant.

Models of the spectral dependence of the solar radiation field from 0.3-4.6 $\mu \mathrm{m}$ have been computed and compared with the measured solar flux profiles. These models have been used to rescale the measured net flux at the Sounder site to account for two effects: (1) the inclusion of the net flux outside of the LSFR passbands and (2) the evaluation of the globally averaged solar net flux profile assuming that the cloud properties at the Sounder site are typical of planetary conditions. It turns out that both these scale factors are reasonably near unity (within $\sim 20 \%$ ) and of opposite sign so the measured net flux profiles need only relatively minor adjustment $(\sim 10 \%)$ to give the bolometric globally averaged solar net flux. Figure 3 shows the globally averaged profile scaled to include solar energy outside the LSFR passband. Also shown are the profiles that result if $2 \%$ relative calibration errors of opposite sign in the upward and downward fluxes plus $10 \%$ absolute errors act together to increase the level of the net flux. Calculations of the net flux profile above $65 \mathrm{~km}$ are also shown for a forward scattering cloud model consistent with the measured spherical albedo of Venus and PV cloud data [Tomasko et al., this issue].

As discussed in the LSFR instrument paper, the measurements indicate that about $17 \mathrm{~W} / \mathrm{m}^{2}$ are absorbed at the ground corresponding to about $2 \frac{1}{2} \%$ of the sunlight incident on the planet. Some $15 \mathrm{~W} / \mathrm{m}^{2}$ are absorbed in the atmosphere below $40 \mathrm{~km}$ altitude. Remarkable little solar energy is absorbed in the dense lower and middle clouds. Indeed, calculations show that the net flux profile is not much affected even for models in which the lower cloud is omitted. By contrast, the upper cloud absorbs solar energy at a much greater rate. About half the solar energy absorbed by Venus is absorbed at altitudes greater than $60 \mathrm{~km}$.

Some checks on this profile can be made. Relative calibration errors in the upward and downward fluxes would cause the net fluxes to vary most at high altitudes and almost not at all at the ground-in effect causing the profile to pivot about the value at the ground. Since the observed profile is quite flat in the lower and middle clouds, relative calibration errors could not decrease the net flux at these levels without causing the net fluxes to increase with decreasing altitude through the clouds - an unphysical result requiring the atmosphere to glow at visible wavelengths. On the other hand, relative calibration errors could not increase the net flux values at middle altitudes $(\sim 30 \mathrm{~km})$ very much without also increasing the net fluxes at $60 \mathrm{~km}$ by so much that the profile would be incompatible with the net solar flux of $\sim 132 \pm 13 \mathrm{~W} / \mathrm{m}^{2}$ measured at the top of the atmosphere [Taylor et al., this issue].

Finally, a comparison of the net solar flux at the ground measured by the LSFR and by the Venera 8-12 entry probes gives some confirmation of absolute calibration and the constancy of the Venus cloud properties. Figure 4 shows the net fux measured at the ground by the LSFR as a little less than $2 \%$ of the incident solar flux at the PV entry site. Venera 8 measured $\sim 1 \%$ at a site much closer to the terminator [ $\mathrm{Av}$ duevsky et al., 1973], while the value given for the Venera 9 and 10 sites is about $3 \%$ at solar zenith angles of $27.7^{\circ}$ and 33.4 [Moshkin et al., 1978]. Moroz et al. [1980] gave a value for the downward flux of $72 \mathrm{~W} / \mathrm{m}^{2}$ at the Venera 11 and 12 sites (solar zenith angle $\sim 20^{\circ}$ ). If the ground reflectivity is as high as $15 \%$, the net flux would be correspondingly lower as indicated by the vertical bar in the figure. The solid curve passing through the PV value shows the variation with solar zenith angle of a forward scattering cloud model. It indicates that the various measurements of solar net flux at the ground fit together remarkably well.

Thermal net flux profiles. The first and only measurements of net thermal radiation in the atmosphere of Venus were made by instruments on the Pioneer entry probes [Boese et al., 1979; Suomi et al., 1979, this issue]. Since results from the large probe instrument were seriously affected below $50 \mathrm{~km}$ by an apparent window misalignment, and are presently being reviewed for possible effects above this level, we will present here only measurements from the small probes.

The small probe measurements were made with an external 
wide-field-of-view radiometer, sensing net radiation over a broad spectral bandpass from $0.2 \mu \mathrm{m}$ to about $150 \mu \mathrm{m}$. The radiometers on the North and Night probes, which entered in darkness, measured profiles of net thermal radiation only. On the Day probe, which entered at a solar zenith angle of $79.9^{\circ}$, the profile includes solar as well as thermal contributions. To determine a thermal net radiation profile for this probe, it is necessary to subtract an estimated solar net radiation profile which can be derived from the LSFR measurements on the Sounder probe and scattering models.

Measured and estimated thermal net flux profiles are presented in Figure 3. The shaded band presented for the Day probe thermal profile is bounded by the estimated uncertainty in the calibration of the LSFR. At the top of each profile, extending over an altitude range of about $5 \mathrm{~km}$, corrected values replace the raw measurements. Two corrections have been applied in this region, both somewhat uncertain at this point [Suomi et al., this issue]: (1) subtraction of an erroneous downard flux induced by the effect of a large thermal transient initiated at deployment together with a small gas flow through the sensor head, and (2) corrections for nonvertical probe orientation. No data are available for altitudes below $12.5 \mathrm{~km}$ because of external sensor failures which occurred on all probes at this level. Night probe data below $27 \mathrm{~km}$ were omitted because of questions raised by the beginning of saturated offset readings at this point.

The probe-to-probe variabilities in the thermal net flux profiles are remarkable and entirely unexpected. Factor of two variations are seen both in the cloud layer (generally above $47-50 \mathrm{~km}$ ) and in the clear region below. Within the cloud layer variations may be attributed to differing cloud properties at the three entry locations, although nephelometer backscatter measurements [Ragent and Blamont, this issue] reveal substantial variations only in the lower cloud layer. Variable amounts of nucleation size haze may be a possible explanation of differences in the upper cloud [Suomi et al., this issue]. In the North probe profile a temperature inversion above $60 \mathrm{~km}$ (apparently typical of high latitudes [Kliore and Patel, this issue] probably contributes to the observed variation. Below the clouds, the apparent absence of significant particulate content and the relative uniformity of thermal structure suggest that compositional variability is required to explain the thermal flux profiles. The structural features seen in the stable layer between the cloud bottoms and about 35 km suggest a fine scale vertical variation of composition as well. Water vapor mixing ratio variations appear to be a possible explanation of these features [Suomi et al., this issue].

Even in the mean, the thermal profiles are unusual. Prior to Pioneer Venus it was expected that solar energy deposition at the surface would exceed thermal losses, maintaining an adiabatic temperature profile by radiative-convective equilibrium. The thermal net flux measurements, on the other hand, indicate that below $13 \mathrm{~km}$ more energy is being lost than is absorbed from the sun. If the mean small probe net fux radiometer (SNFR) fluxes were representative of the planetwide average, the implied rate of energy loss would be large, between 15 and $30 \mathrm{~W} / \mathrm{m}^{2}$. This loss is not consistent with the most recent radiative transfer calculations (described in section 4), and should not be trusted until the possibility of SNFR measurement errors in the lower $35 \mathrm{~km}$ is more completely investigated.

The radiative gains and losses within the atmosphere due to thermal (planetary) radiation are indicated by the vertical de- rivative of the net fluxes; a positive slope implies cooling, a negative slope heating. A consistent feature seen in all the thermal profiles is a radiative heating at the base of the lowest significant cloud. This would tend to support the higher temperatures (relative to adiabatic extrapolations from below) measured at the cloud base and contribute to stabilization of the atmosphere below the clouds and destabilization immediately above cloud base. Except for the possibility of significant cooling below $12.5 \mathrm{~km}$, where no thermal measurements are available, the bulk of the atmospheric thermal loss (and certainly the greatest cooling rate) occurs near the cloud tops. This is most apparent in the Night probe profile, apparently a result of the unusually low cloud top altitude at this location [Ragent and Blamont, this issue]. In general, however, the net thermal fluxes measured by the probes at the highest altitudes do not provide an estimate of the thermal loss to space because of significant gas and particulate opacity (especially for the North and Day probes) above the earliest measurements.

\section{Thermal Opacity Sources}

Cloud particles. The Pioneer Venus mission has returned a wealth of data bearing on the physical and optical properties of the ubiquitous cloud layers. Knollenberg et al. [this issue] summarize the results from the relevant experiments. It is impressive how much information is now available concerning the cloud particles and yet the composition of at least some of the particles is still unknown. Furthermore, the number density of the submicron particles, below the minimum size limit of the cloud particle size spectrometer (LCPS), remains to be measured. Despite the remaining uncertainties, the cloud particle measurements provide valuable information concerning the infrared opacity of the clouds.

At the Sounder probe entry site the LCPS measured the number density of the cloud particles as a function of the size [Knollenberg and Hunten, this issue]. This experiment is able to define the shapes of two size distributions, one with a mean diameter of 2.1-2.7 $\mu \mathrm{m}$ called mode 2 and another with mean diameter of about $7 \mu \mathrm{m}$ called mode 3 . A third size mode (mode 1$)$ is found in the smallest size bin (0.6-1.1 $\mu \mathrm{m}$ diameter) but the mean diameter of the mode is unknown from this experiment. However, the cloud photopolarimeter (OCPP) experiment does have information from high altitude hazes which defines a size mode that is consistent with mode 1 in the cloud deck [Kawabata et al., this issue].

The Venus clouds are stratiform and found in three major layers with haze regions both above and below the main cloud layers. The upper haze containing only mode 1 is found above about $65 \mathrm{~km}$. This layer seems to be located primarily in the polar region beyond about $50^{\circ}$ latitude. It is also seen in an attenuated form at lower latitudes concentrated near the terminators. The upper cloud region (57-65 km) is apparently a global feature. It has a bimodal size distribution as seen from both the orbiter and the LCPS (modes 1 and 2). A large fraction of the incoming sunlight is absorbed in this region, which implies that one or both of the modes has a low single scattering albedo. This is inconsistent with a composition of only concentrated sulfuric acid. Absorption in the ultraviolet portion of the spectrum is due to a combination of materials whose identity is uncertain. A discussion of the various candidates for this absorption is given in Pollack et al. [this issue].

The mode 2 distribution in this upper cloud region has a modal diameter of about $2.1 \mu \mathrm{m}$ consistent with earth-based polarimetry analyses of Hansen and Hovenier [1974]. These 


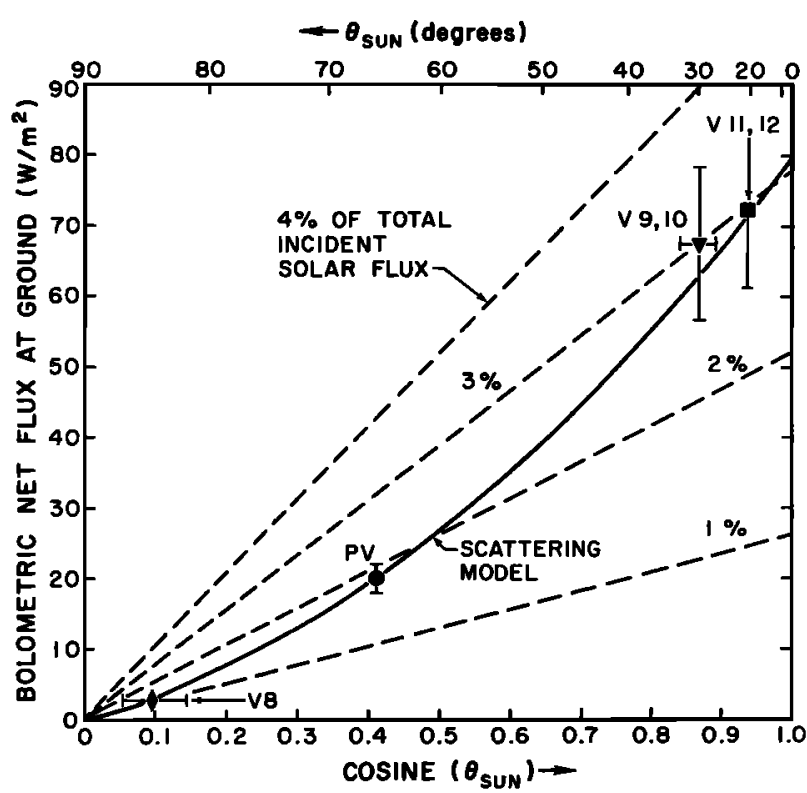

Fig. 4. Net solar flux at the surface of Venus as a function of the cosine of the solar zenith angle. Dashed lines represent constant fractions of the incident solar flux as indicated. The solid line represents the variation computed for a forward scattering cloud model adjusted to pass through the Pioneer Venus measurement at the Sounder probe site (labeled PV). This measurement and those measured at the Venera 8 (labeled V8), Venera 9 and $10(\mathrm{~V} 9,10)$ and Venera 11 and $12\left(V_{11}, 12\right)$ sites are quite consistent as discussed in the text.

authors were able to establish the cloud particles as spherical with a narrow size distribution and a refractive index of 1.44 at $0.55 \mu \mathrm{m}$. The combined LCPS and nephelometer measurements confirm these results. This mode is firmly identified with concentrated sulfuric acid.

For any size mode assumed to consist of spherical $\mathrm{H}_{2} \mathrm{SO}_{4}$ particles, the refractive indices measured by Palmer and Williams [1975] and Mie theory can be used to compute the ratio of the absorption cross section to the visible extinction cross section (shown in Figure 5 as a function of wave number). A blackbody curve for $270 \mathrm{~K}$, the temperature below the upper cloud deck, is shown for comparison. Since the visible optical depth for mode 2 in the upper clouds is about 6 , the infrared optical depth due to this mode can be estimated as $6 \times 0.05=$ 0.3 in the $20-100 \mu \mathrm{m}$ region. The additional opacity due to mode 1 can be expected to be even less. Therefore on the basis of PV cloud particle measurements, the upper cloud region will be optically thin in the thermal infrared.

The lower and middle cloud regions are trimodal containing an additional mode of larger particles (mode 3 ). The mean diameter of mode 2 shifts abruptly below the upper cloud layer to $2.7 \mu \mathrm{m}$ (it is now referred to as mode $2^{\prime}$ for clarity). Figure 5 shows modes $2^{\prime}$ and 3 versus wave number compared to a blackbody curve for $T=380 \mathrm{~K}$, the temperature below the lower cloud. Mode 3 would be the dominant source of thermal opacity in these two regions assuming a sulfuric acid composition. However, Knollenberg and Hunten [this issue] present strong evidence that the mode 3 particles are crystalline; various sulfates and chlorides and their hydrates are suggested. In addition, the solar net flux profile constrains these particles to be essentially conservative scatterers at visible wavelengths [Tomasko et al., 1979, this issue] and the SNFR net fluxes show their thermal opacity is significantly smaller than that for spherical $\mathrm{H}_{2} \mathrm{SO}_{4}$ particles. To model the radia- tive properties of the mode 3 cloud, we assume the variation with frequency of the ratio of thermal to visible opacity is that given in Figure 5 for liquid $\mathrm{H}_{2} \mathrm{SO}_{4}$, but we decrease the effective visible optical depth of the mode 3 particles to be consistent with the LSFR solar flux profiles in order to simulate the smaller opacity expected for irregular crystals. The resultant decrease in thermal opacity for this mode is also approximately consistent with small probe net flux measurements. Below the lower cloud no appreciable infrared opacity due to aerosols is expected from the very thin haze of small particles seen down to $35 \mathrm{~km}$ altitude.

Gaseous opacity. In evaluating the infrared opacity of the gases present in the atmosphere of Venus, we can make use of both the Pioneer Venus and the Venera compositional measurements. For the most part we have used the results of Hoffman et al. [this issue] and Oyama et al. [this issue] with the exception of the $\mathrm{H}_{2} \mathrm{O}$ abundance which we discuss briefly below. To illustrate the spectral dependence of opacity and the relative contributions of different gases, we consider a $1 \mathrm{~km}$ thick layer of the atmosphere at the 20 bar level.

The major infrared active gas is, of course, carbon dioxide. We find from the room temperature laboratory data of $\mathbf{R}$. W. Boese and P. M. Silvaggio (private communication, 1979) that at 20 atm this gas is essentially opaque over the $450-2550$ $\mathrm{cm}^{-1}$ spectral region. There are two transmission window regions from approximately 1100 to $1200 \mathrm{~cm}^{-1}$ and from 1600 to $1900 \mathrm{~cm}^{-1}$. Deeper in the Venus atmosphere, at higher temperatures and pressures, we would expect these windows to be closed or partially closed.

One of the exciting findings of the Pioneer Venus mission was that sulfur dioxide is approximately 1000 times more abundant than deduced from remote observations. The absorption intensity of many of the infrared bands of this molecule have not yet been measured. However, a fundamental vibration band (which has been measured) is centered at 1151 $\mathrm{cm}^{-1}$. Considering the reported abundance of $\mathrm{SO}_{2}$, this band will easily close the $1100-1200 \mathrm{~cm}^{-1}$ carbon dioxide window. In addition, there are at least three overtone/combination $\mathrm{SO}_{2}$ bands in the $1600-1900 \mathrm{~cm}^{-1}$ window region (intensities unmeasured) which will probably close this window.

The far-infrared absorption by $\mathrm{SO}_{2}$ has recently been measured by R. S. Larkin et al. (private communication, 1980); that of $\mathrm{CO}_{2}$ has been reported by Birnbaum et al. [1971]. These measurements indicate that, at the 20 bar level, the atmosphere of Venus is opaque from $10 \mathrm{~cm}^{-1}$ to at least 120 $\mathrm{cm}^{-1}$. Thus these two species alone blanket most of the infrared spectral region. The remaining windows are from about 22 to $80 \mu \mathrm{m}$ and shortward of about $2.5 \mu \mathrm{m}$.

Of the atmospheric species detected by the Pioneer Venus mission [Hoffman et al., this issue] three may help to close the long wavelength window; $\mathrm{H}_{2} \mathrm{~S}, \mathrm{C}_{2} \mathrm{H}_{6}$, and $\mathrm{COS}$. However, their low abundance values dictate that they cannot be the sole opacity source. The greenhouse must rely on water vapor absorption for this region and some other opacity source at short wavelengths.

The abundance of water vapor reported by the Pioneer Venus mission is approximately a factor of 30 larger than deduced by the Venera mission. If the water vapor abundance reported by Oyama et al. [this issue] is correct, there certainly is sufficient opacity for the atmospheric greenhouse. However, Moroz et al. [1980] report water abundances of $\sim 20$ ppm near the surface and $\sim 200 \mathrm{ppm}$ in the clouds. In this case the calculations of the thermal net flux in the $22-80 \mu \mathrm{m}$ region may de- 


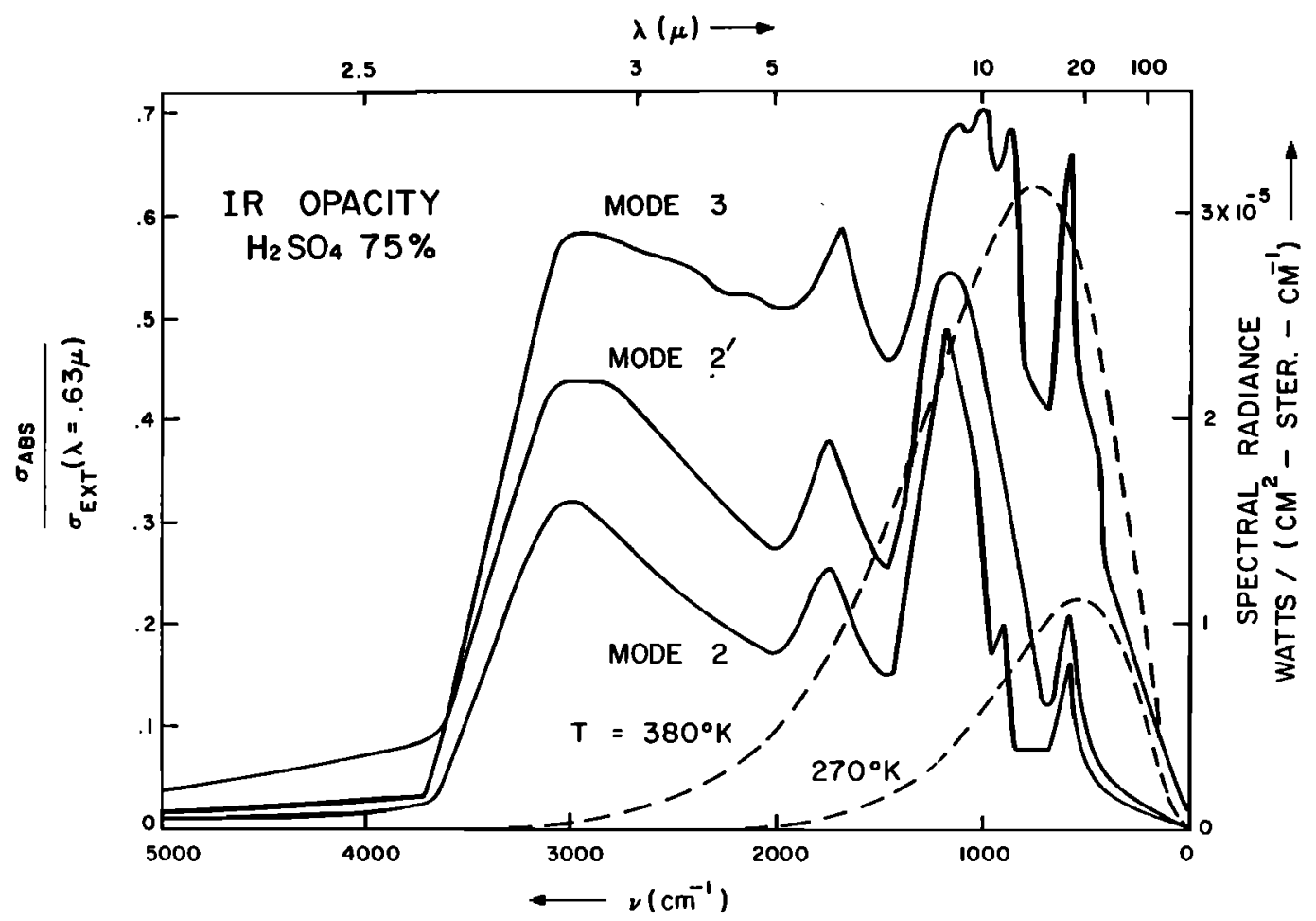

Fig. 5. The ratio of infrared absorption cross section to extinction cross section in the visible $(0.63 \mu \mathrm{m})$ as a function of wavelength for sulfuric acid droplets having the size distributions measured for size modes $2,2^{\prime}$, and 3 by the cioud particle size spectrometer (see text). Also shown are the blackbody emission curves (dashed lines) for $270 \mathrm{~K}$ and $380 \mathrm{~K}$, the temperatures at the base of the upper and lower cloud layers, respectively, on Venus.

pend sensitively on the exact water vapor abundance and our knowledge of the behavior of the water vapor bands in this spectral interval at the elevated temperatures and pressures found on Venus.

\section{INTERPRETATION OF THE \\ RADIATION MEASUREMENTS}

Thermodynamic Constraints on the Net Flux Profiles

The large thermal net fluxes measured at low altitudes were so unexpected that the constraints placed on the net flux profiles by the laws of thermodynamics have been investigated [Ingersoll and Pechmann, this issue; Suomi et al., this issue]. Constraints on the flux profile follow from the assumption of a steady state atmosphere in which the total rate of change of entropy due to solar energy absorption $\boldsymbol{S}_{s}$ (positive), that due to thermal radiative exchanges $S_{p}$ (negative), and that due to internal dissipative effects $S_{d}$ (positive) must sum to zero. This leads to the inequality $\left|S_{p}\right|>S_{s}$ which implies that solar and thermal radiative exchanges have different distributions within the atmosphere, so that, on the average, thermal losses occur at a lower temperature than that at which solar energy is absorbed.

Suomi et al. [this issue] have shown that the mean of the entropy change from planetary radiation $S_{p}$ is just about equal to the entropy change for the 'high solar' profile shown in Figure $3\left(\sim 0.48 \mathrm{~W} / \mathrm{m}^{2}-\mathrm{K}\right)$. A more stringent bound on the difference $\left|\dot{S}_{p}\right|-\boldsymbol{S}_{s}$ can be obtained by estimating the entropy production by convective heat transfer and dissipation of mechanical energy.

Ingersoll and Pechmann [this issue] argue that including the convective flux leads to an entropy production rate too high for steady state thermal models of the atmosphere of Venus unless some of the flux profiles are incorrect, or unrepresentative, of globally averaged conditions. However, the values obtained for the solar and thermal entropy intergrals depend sensitively on the shape of the flux profiles adopted between 60 and $90 \mathrm{~km}$ altitude - a region where only model calculations are currently available. As data from the PV orbiter continue to be analyzed, the shape of the solar and thermal net flux profiles above the clouds should be better known. If the new data indicate a net generation of entropy, several possible resolutions of this problem can be considered as suggested by Ingersoll and Pechmann [this issue] and by Suomi et al. [this issue]. With the current uncertainties in the flux profile, detailed consideration of these suggestions is not warranted at this time.

\section{Role of Radiative Processes in Vertical Energy Transfer}

We begin consideration of the role played by radiative processes in the energy balance of the atmosphere by comparing the measured thermal net fluxes with the fluxes computed for several models of the atmosphere. The nominal model is based on the PV measured state parameters, cloud densities and size distributions. The optical depth of the mode 3 cloud particles is about $40 \%$ of Knollenberg and Hunten's [1979] published value to take into account the ncn-spherical aspect ratio as discussed earlier in section 3. The water mixing ratio is taken from Venera 11 and 12 measurements [Moroz et al., 1980]. For the effect of other minor gaseous absorbers see Pollack et al. [1980].

Cloud regions and above. A comparison of the net fluxes measured by the small probe net flux radiometers (SNFR) [Suomi et al., this issue] and the predicted net fluxes from the 


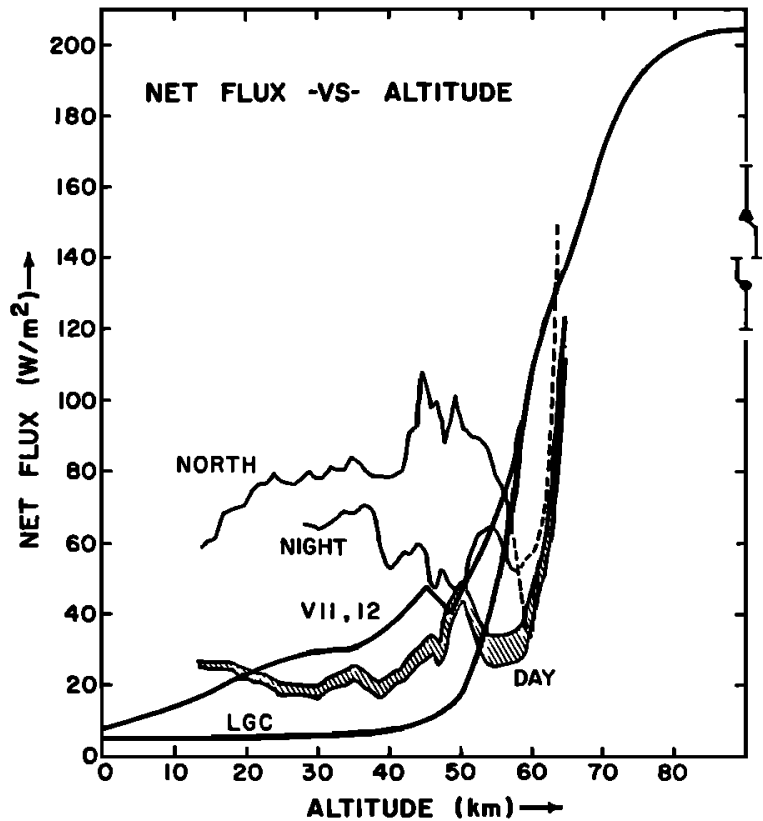

Fig. 6. Thermal net fluxes predicted by the nominal atmosphere model are compared to the net flux profiles measured by the three SNFR's. The model predicts that $205 \mathrm{~W} / \mathrm{m}^{2}$ is emitted to space compared to the value measured by the OIR (triangle) and the Bond albedo (circle). While the nominal model contains the water vapor abundances measured by Venera 11 and $12(\mathrm{~V} 11,12)$, the model has been rerun with the considerably larger $P V$ water vapor abundances (LGC) for comparison.

nominal model atmosphere is shown in Figure 6. The nominal model predicts that the atmosphere as a whole is losing energy by thermal radiation to space at a rate of about $205 \mathrm{~W} / \mathrm{m}^{2}$, in excess of the OIR measurement of $153 \pm 13 \mathrm{~W} / \mathrm{m}^{2}$ [Taylor et $a l .$, this issue]. The emission to space predominately originates from the vicinity of the cloud tops, i.e., the upper cloud region. It is here that another thermal opacity source must be included in order to reproduce the upper boundary condition. This conclusion is bolstered by the surplus of net flux predicted at $60 \mathrm{~km}$ to that measured by the SNFR. A third and independent indication of problems in this region is the necessity of obeying the entropy constraint discussed above. The largest contribution to the entropy integral comes in this altitude range. If the altitude of optical depth unity for thermal emission is much lower than that for solar absorption, the atmosphere absorbs energy at a lower temperature than that at which it radiates to space thus violating the entropy constraint.

The addition of increased gaseous, or aerosol, opacity sources to this region of the atmosphere can be considered. A simple method of estimating the amount of trace gas necessary to meet the boundary condition is to add water to the upper cloud level (below $\tau \geq 1$ since $\sim 1 \mathrm{ppm}$ is measured from the earth). Model atmospheres with enhanced water profiles have been tried, but they require unrealistic mixing ratios far in excess of the saturation vapor pressure. Pollack et al. have computed greenhouse models which include $\mathrm{SO}_{2}, \mathrm{HCl}$, and $\mathrm{CO}$ in addition to water. After conducting a series of sensitivity calculations they conclude that $\mathrm{SO}_{2}$ is indeed of secondary importance to the thermal balance problem (where $\mathrm{HCl}$ and $\mathrm{CO}$ are of tertiary importance). However, they cannot match the observed lapse rate in the upper cloud region with even 10 times the nominal water plus $\mathrm{SO}_{2}$. Therefore the dominant source of opacity is most likely aerosols.
Overall, there is remarkable agreement concerning the properties of the upper cloud particles from the LCPS, the OCPP, the nephelometers and earth-based polarization measurements. Larger particles cannot be present in any sizable proportion without having been observed by these instruments. One remaining option (suggested by Suomi et al. [1979, this issue] is the possibility that submicron particles much smaller than the nominal mode 1 distribution are present in sufficient number density to offer IR opacity.

Since the Mariner 10 mission a strong case for the presence of submicron aerosols in the upper cloud and haze layers has been developed from observations in the UV, visible and near infrared [cf. O'Leary, 1975; Martonchik and Beer, 1975; Lane, 1979]. None of these authors have discussed the important role that these haze layers might play in regulating the thermal balance of the Venus atmosphere. For the purposes of this discussion we will call these particles 'mode 0' to distinguish them from the larger mode 1 particles.

Laboratory studies of the nucleation of $\mathrm{H}_{2} \mathrm{SO}_{4}$ particles from $\mathrm{SO}_{2}$ gas in air irradiated by simulated solar UV fluxes show that one can expect large quantities of submicron particles in proportion to the abundance of $\mathrm{SO}_{2}$ and the relative humidity $h$ [Shen and Springer, 1976]. The relation observed by these authors is

$$
N=3.33 \times 10^{6}\left(\mathrm{SO}_{2}\right)(h)^{2} \mathrm{~cm}^{-3}
$$

where $\left(\mathrm{SO}_{2}\right)$ is in ppm and $h$ is in percent. For $\left(\mathrm{SO}_{2}\right)$ of $0.1-10$ ppm and $h$ of $0.5-1 \%$, the approximate Venus upper cloud environment, this expression predicts $N \sim 10^{5}-10^{7} \mathrm{~cm}^{-3}$. The quantity $N$ in this equation is for particles with diameters $<0.1$ $\mu \mathrm{m}$. No particles with diameters greater than $0.3 \mu \mathrm{m}$ were measured in the laboratory after UV irradiation times of several minutes although the number density of small particles has asymptotically approached a maximum. In addition, earth-based atmospheric studies [cf. Whitby, 1978] reveal the existence of a nucleation mode of large number density with a mean diameter of about $0.01 \mu \mathrm{m}$ as well as an accumulation mode which has a mean diameter of $0.069 \mu \mathrm{m}$. Therefore it seems likely that these modes must exist in some form in the Venus atmosphere at altitudes fulfilling the proper conditions of UV flux, $\mathrm{SO}_{2}$ abundance and relative humidity. Just the right conditions are found in the upper cloud and in the upper haze. In the middle cloud the UV radiation has been largely absorbed out of the spectrum.

To briefly outline the characteristics mode 0 must have to be an important infrared opacity source let us assume that the haze has a constant number density and extends above the middle cloud $(57.5 \mathrm{~km})$ for $30 \mathrm{~km}$. The optical properties of such an acid haze are markedly different from the visible to the infrared. The particles are conservative scatterers in the visible with a real index of about 1.43; however, beyond 2.8 $\mu \mathrm{m}$ they become strongly absorbing with an imaginary index approaching 1.0. The infrared optical depth (due almost entirely to absorption) is a function of the mass loading for a fixed imaginary index. The visible (scattering) optical depths depend on the size of the particles in which the mass is placed. Therefore the ratio $R$ of the absorption optical depth in the infrared to the extinction optical depth in the visible $(0.63 \mu \mathrm{m})$ can be large for sufficiently small particles which have a large imaginary index in the infrared and a small imaginary index in the visible. The upper limit on the effective radius of the particles will be set by determining how much scattering optical depth can be tolerated in the visible and UV. A lower limit on the effective radius is set by the condition that coagulation 
does not cause the particles to grow out of the required size range faster than they can be replaced.

As an illustration, we will select a mass loading which gives an infrared optical depth of 1.0 at $10 \mu \mathrm{m}$. In order to get an average particle lifetime of several hours, we choose a narrow mode distribution centered at $0.035 \mu \mathrm{m}$ with a total number density of $2 \times 10^{5} \mathrm{~cm}^{-3}$. The $R$ value is about 17.5 so that the visible optical depth is negligible at 0.06 ; however, note that the particles are Rayleigh scatterers so that the scattering optical depth at $0.32 \mu \mathrm{m}$ wavelength will be near unity. Larger particles will substantially increase the UV optical depth for a constant mass loading of $0.03 \mathrm{mg} / \mathrm{m}^{3}$. Thus the two consideration of UV optical depth and long lifetime place tight constraints on the choice of an effective radius for the mode 0 particles.

Even though the thermal opacity of mode 0 decreases linearly to zero with decreasing frequency, model calculations indicate that it can be successful in lowering the calculated thermal flux at the top of the atmosphere by $50 \mathrm{~W} / \mathrm{m}^{2}$ so that it is consistent with the orbiter measurements described earlier. While we have not explored the cloud physics of these small particles to any great extent, Toon et al. [1979] find that particles of approximately this size range, number density, and altitude distribution are a natural consequence of their physical-chemical model of the Venus clouds. Their model also reproduces the measured cloud properties above $58 \mathrm{~km}$.

Region below the clouds. Prior to Pioneer Venus, the greenhouse effect represented the most popular explanation for Venus' high surface temperature [Sagan, 1960; Pollack, 1969]. According to this model, the atmosphere is more transparent in the visible than in the infrared region of the spectrum. As a result, sunlight more easily penetrates to the surface than thermal radiation from the surface can escape to space. Because the surface temperature of Venus is elevated by about $500 \mathrm{~K}$ above the planet's effective temperature, the atmosphere must have considerable opacity at all thermal wavelengths, in contrast to the situation for the earth, where several major 'window' regions occur.

In part, acceptance for the greenhouse explanation of Venus' surface temperature can be attributed to the apparently reasonable requirements it placed on the abundances of the major infrared absorbers. In the classical models, $\mathrm{CO}_{2}$ and $\mathrm{H}_{2} \mathrm{O}$ were assumed to be the key absorbers, with about $1 / 2 \%$ of $\mathrm{H}_{2} \mathrm{O}$ vapor by volume being required in the lower atmosphere [Pollack, 1969]. As results from the early Venera spacecraft probes into Venus' atmosphere became available, it was realized that the optically thick sulfuric acid clouds could also make a significant contribution to the greenhouse effect [Pollack and Young, 1975].

The most serious alternative model was one in which the large scale atmospheric dynamics created the high surface temperature [Goody and Robinson, 1966]. According to this model, heat was vertically advected downward by the general circulation from the cloud region, where all the solar energy was assumed to be deposited, to the lower atmosphere and surface. Temperature gradients between the subsolar and antisolar point drove the circulation. However, when allowance was made for the density stratification of the atmosphere, numerical circulation models failed to achieve the desired surface warming [Kálnay de Rivas, 1975].

Initially, the PV thermal flux measurements and the Venera 11 and $12 \mathrm{H}_{2} \mathrm{O}$ mixing ratio estimates suggested that the atmosphere of Venus was much less opaque than expected. However, Pollack et al. [this issue] have carried out a new series of

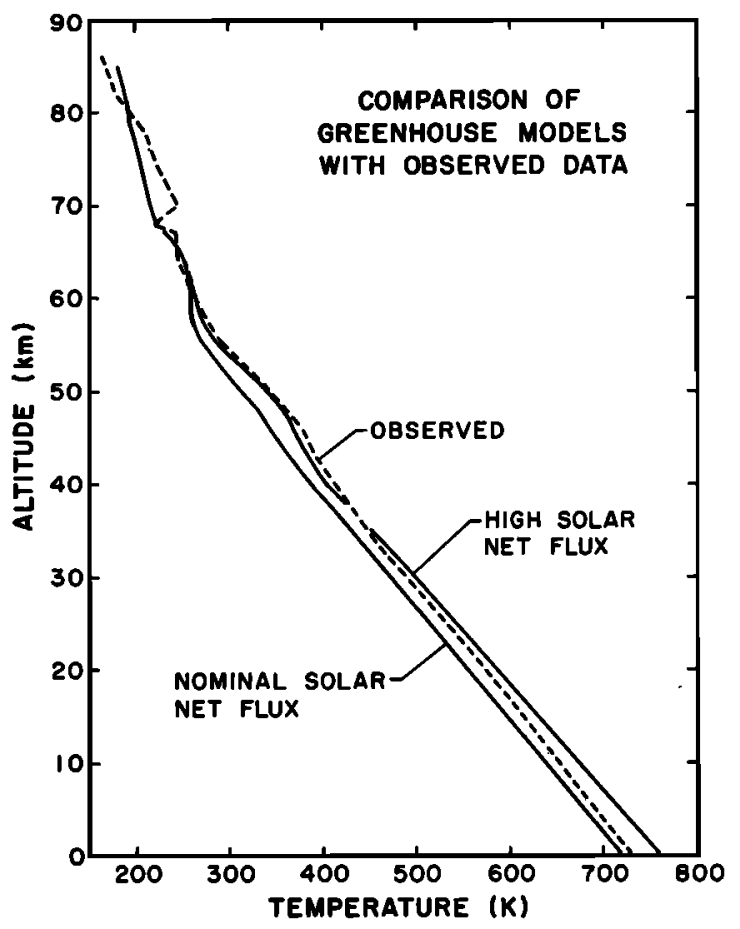

Fig. 7. A one-dimensional radiative-convective greenhouse model is shown with the temperature profile adjusted such that the thermal net fluxes equal the solar net fluxes. Wherever the calculated lapse rate exceeds the adiabatic lapse rate, it is assumed that convective processes dominate. The opacities are taken from the nominal model plus enough mode 0 aerosols in the upper cloud region and above to match the observed effective temperature. Both the nominal and the high solar net flux profiles are tried and compared with the temperature profile observed by PV at the large probe site (see Figure 1).

greenhouse calculations incorporating enhanced $\mathrm{CO}_{2}$ opacities based on laboratory measurements of pressure induced transitions. Using Moroz's water vapor profile and incorporating mode 0 particles into the upper portion of the clouds, they obtained values for the surface temperature of $720 \mathrm{~K}$ and 760 $K$ with the 'nominal' and 'high' global solar net flux profiles of Tomasko et al. [this issue], respectively. These predicted values bracket the observed value of $730 \mathrm{~K}$. In addition, the temperature stability structure of these models reproduced approximately the observed structure in the lower atmosphere $(0-65 \mathrm{~km})$. Figure 7 illustrates the temperature profile of these two models and compares them with the observed profile of Seiff et al. [1979b] at the location of the Pioneer Venus Sounder probe. The success of these model calculations suggests that almost all of the high surface temperature of Venus is due to a very effective greenhouse.

There are several additional aspects of the above calculations that warrant comment. First, results obtained with the greatly enhanced water vapor abundances ( 2000 ppm) reported by the Pioneer Venus gas chromatograph were much less satisfactory than those obtained with the Venera spectrophotometer abundances, particularly with regard to reproducing the stably stratified region located immediately below the main clouds. Second, while the inclusion of the mode 0 particles led to only a small enhancement of the surface temperature $(\sim 30 \mathrm{~K})$, they exerted a strong influence on the stability structure both within the clouds and below them, with the resulting structure being sensitive to their altitude profile. Finally, the dominant sources of infrared opacity in the nominal models were $\mathrm{CO}_{2}, \mathrm{H}_{2} \mathrm{O}$, cloud particles, and $\mathrm{SO}_{2}$, in order of decreasing importance. 
For the most part, the temperature profiles obtained below $35 \mathrm{~km}$ altitude in the greenhouse calculations are not too sensitive to increases in the opacity tables because they already require lapse rates as steep as the adiabatic value at these levels. Nevertheless, the calculations of net thermal flux are hampered by lack of complete opacity data of $\mathrm{CO}_{2}, \mathrm{H}_{2} \mathrm{O}$, and $\mathrm{SO}_{2}$ at high temperature, pressure, and abundance. At least some authors [Young, 1979] maintain that a comprehensive analysis of $\mathrm{CO}_{2}$ alone at the elevated temperatures and pressures of the lowest $20 \mathrm{~km}$ of the Venus atmosphere would reveal large opacities in several of the spectral regions commonly considered to be $\mathrm{CO}_{2}$ windows such as between 8-11 $\mu \mathrm{m}$ and shortward of $2.5 \mu \mathrm{m}$. It is fair to say that there is some doubt that larger thermal net fluxes can be maintained in the lower Venus atmosphere.

Thus even calculations using recently revised opacity data [Pollack et al., this issue] may be incomplete and tend to provide an upper limit to the fluxes likely to be present in the lower atmosphere. The direct measurements of the net thermal flux in the lower atmosphere significantly exceed these calculated estimates as shown in Figure 6. The North and Night probe fluxes in particular are so great below $35 \mathrm{~km}$ that instrumental problems are suspected. To date, although no instrumental problem has been identified, there are certain descent conditions which may perturb flux measurements in the deep atmosphere but which have not yet been adequately simulated in laboratory tests [Suomi et al., this issue]. Work in this area is continuing.

\section{Role of Dynamics in Maintaining ThERMal Balance}

A general discussion of the structure and circulation of the Venus atmosphere in light of all Pioneer Venus results is given by Schubert et al. [this issue]. Here we briefly indicate the PV results concerning atmospheric circulation and discuss the role of dynamical processes in maintaining the thermal balance of the Venus atmosphere.

The relationship between radiative energy exchanges and the circulation of an atmosphere is a complex feedback process in which radiation creates small horizontal gradients of the atmospheric state parameters that force circulation. In turn, the circulation affects the size of the state parameter gradients and the radiative exchanges. On Venus, this process has yielded an atmosphere in which the horizontal variability of pressure, temperature, and even composition is small by earth standards. Since the radiative energy deposition from the sun is not uniform over the planet, dynamical energy transport is required if the present state of the atmosphere is to be maintained.

Circulation patterns have been measured in some portions of the Venus atmosphere which could provide the energy transport needed to explain the lack of horizontal structural variability over parts of the planet. The dominant circulation at cloud levels is a zonal retrograde motion. Pioneer Venus has confirmed earlier observations that the motion is roughly equivalent to a superrotation of the upper atmosphere, with a period of about 4 earth days at cloud top heights [Counselman et al., this issue; Rossow et al., this issue; Seiff et al., this issue]. The superrotation must be a consequence of the radiative heating distribution, but the actual mechanism for driving the rapid zonal winds is unclear at present. Proposed explanations for the superrotation have relied on motions induced directly or indirectly by radiative heating [Young and Schubert, 1973;
Gierasch, 1975; Young and Pollack, 1977]. Thus in attempting to understand the drive behind the rapid zonal circulation, it is important to look for correlations between the flux profiles obtained by the LSFR and SNFR and the zonal wind profiles. The $30 \mathrm{~km}$ altitude level divides the Day and Night SNFR profiles into two distinct regions: a lower one of uniform radiative heating and an upper one with a complicated structure of alternating heating and cooling [Suomi et al., 1979, this issue]. This level also divides the Day and Night zonal wind profiles [Counselman et al., this issue] into two distinct parts [Schubert et al., this issue]. In the lower portion of the "stair-step" structure velocities reach about $25 \mathrm{~m} \mathrm{~s}^{-1}$; in the upper part velocities build up to over $100 \mathrm{~m} \mathrm{~s}^{-1}$. Comparisons among the simultaneous measurements of zonal wind velocity by the four Pioneer Venus probes are another way to provide constraints on the mechanism by which radiative heating drives the 4-day circulation. In particular, the very close similarity between the Day and Night probe zonal wind profiles [Counselman et al., this issue] suggests that the processes driving the super-rotation are not confined to the region of the subsolar point, but occur everywhere over the planet.

In addition to small diurnal temperature variations, the observed equator-to-pole temperature gradient is quite small as seen in the direct measurements [Seiff et $a l ., 1979 a, b$, this issue; Taylor et al., 1979b, c; Kliore and Patel, this issue; Schubert et al., this issue], and observations of upward infrared flux by the Night and North probes. The latter indicate that there is little variation of flux with latitude up to $\sim 60 \mathrm{~km}$ altitude, the limit of measurement [Suomi et al., 1979, this issue]. Since the infrared cooling of the planet varies more slowly with latitude than does the solar heating (see Figure 2) some form of dynamical transport of heat from equator to pole must be occurring.

The simplest way in which heat could be dynamically transported from equator to pole is by a direct Hadley circulation. This would involve rising motion over the equator, poleward motion at and above cloud top heights, sinking over the pole, and an equatorward return flow at lower levels. There is some direct evidence for just such a Hadley circulation at cloud levels [Schubert et al., this issue]. Meridional winds deduced from the tracking of Pioneer Venus probes are generally equatorward between about 55 and $30 \mathrm{~km}$ altitude [Counselman et al., 1979, this issue; Seiff et al., this issue], while poleward motion is indicated by the tracking of cloud features at cloud top (60-70 km) altitudes [Suomi 1974; Rossow et al., this issue]. Furthermore, the greatly reduced infrared cloud opacity near the north pole is probably caused by atmospheric subsidence at high latitudes [Taylor et al., 1979a].

However, it must be emphasized that a Hadley circulation is not the only way an atmosphere can transport heat poleward. On earth, for example, a Hadley circulation exists between the equator and about $\pm 30^{\circ}$ latitude, but poleward heat transport in the mid-latitudes is accomplished by baroclinic waves [see, e.g., Holton, 1972]. It is possible that in the Venus atmosphere wave or eddy motions also play an important role in meridional heat transport at some latitudes. The "polar collar" of high clouds detected by the orbiter infrared radiometer [Taylor et al., 1979b, c] does in fact suggest a change of circulation regime at high latitudes, but there is as yet no direct evidence to support this concept.

In addition to the planetary-scale circulations discussed above, radiative heating can drive small and intermediate 
scale motions such as cellular convection. A necessary condition for the occurrence of convection is that the decrease of temperature with height be large enough to make the static stability of the atmosphere vanish. Pioneer Venus observations indicate that this occurs in two altitude ranges: the middle cloud level ( $50-55 \mathrm{~km})$ and the lower atmosphere (below $25 \mathrm{~km}$ ) [Seiff et al., 1979a, this issue]. Significantly, the Day probe SNFR measured net radiative heating in both these regions. This suggests that convective motions are driven at these levels. Some evidence exists to support this possibility. Preliminary analysis of probe accelerometer data indicates vertical winds of $\sim 0.5 \mathrm{~m} \mathrm{~s}^{-1}$ between about $25 \mathrm{~km}$ altitude and the surface [Seiff et al., this issue]. Pioneer Venus ultraviolet images show cellular structures (with diameters of a few hundred kilometers) near and downwind of the subsolar point [Travis et al., 1979; Rossow et al., this issue]. These features are reminiscent of mesoscale convection cells seen in weather satellite pictures of the Earth [Agee et al., 1973].

Thus far we have concentrated our discussion on the levels of the atmosphere where most of the solar heating and cooling takes place-the cloud levels and above. Beneath this region there is an atmosphere some 100 times as massive as the earth's. Very little is known about the circulation below the clouds except that the motions are relatively slow. The meridional wind velocities drop below measurement uncertainties of $1-2 \mathrm{~m} \mathrm{~s}^{-1}$ at altitudes lower than about $30 \mathrm{~km}$, and the zonal winds fall to zero (to within measurement uncertainty) below $10 \mathrm{~km}$ altitude [Counselman et al., this issue]. In the lowest few scale heights, the huge thermal inertia of the deep atmosphere suppresses diurnal temperature variations even in the absence of a zonal circulation [Stone, 1975]. The nature of the mean meridional circulation below the clouds is speculative. Schubert et al. [this issue] discuss several possibilities including cells stacked one upon the other, with some of the cells involving direct thermally driven flows while others undergo frictionally driven indirect motions. While some combination of direct and indirect multiple cellular structure might exist in Venus' atmosphere, the net dynamical heat transport would have to be poleward.

The PV measurements of winds, temperature contrasts, and solar and thermal fluxes obtained globally above the clouds and at the probe sites below the clouds have not yet been incorporated into a comprehensive circulation model for Venus. While the one dimensional radiative-convective calculations discussed in section 4 have been very instructive, until such a comprehensive model of the circulation of the atmosphere of Venus is constructed, our understanding of its thermal balance will remain incomplete. The task of determining and understanding the circulation patterns and the advection of heat in the atmosphere of Venus within the constraints imposed by the recent measurements still requires considerable effort before being realized.

\section{SUMMARY AND CONCLUSIONS}

Our main conclusions regarding the thermal balance of the atmosphere of Venus can be summarized as follows.

1. The globally averaged effective temperature for thermal radiation is measured by $P V$ as $228 \pm 5 \mathrm{~K}\left(153 \pm 13 \mathrm{~W} / \mathrm{m}^{2}\right)$ consistent with earlier measurements. The Bond albedo has been measured as $0.80 \pm 0.02$ (implying the absorption of 132 $\pm 13 \mathrm{~W} / \mathrm{m}^{2}$ of sunlight) also consistent with earlier data. The uncertainties of these preliminary results are too large to interpret them as evidence for an internal energy source.
2. The thermal opacity of cloud particles having the properties directly measured by the Sounder probe and $\mathrm{CO}_{2}, \mathrm{H}_{2} \mathrm{O}$ and $\mathrm{SO}_{2}$ is not sufficient to prevent the thermal emission of $\sim 200 \mathrm{~W} / \mathrm{m}^{2}$ to space by the planet. The presence of a mode of submicron aerosols in the vicinity of the upper cloud with modest mass loading would reduce the flux loss to the observed value. These particles would also be important in making the planetary emission occur at slightly higher altitudes (lower temperatures) than those at which solar radiation is absorbed as required by thermodynamic arguments.

3. The measured solar flux profile indicates that about half of the solar energy absorbed by Venus is absorbed above $60 \mathrm{~km}$ altitude. Scattering models indicate PV measurements of solar net flux at the ground are in agreement with Venera 8-12 measurements and show that $\sim 2 \frac{1 / 2}{2}$ percent of the total solar energy incident on Venus is absorbed at the ground.

4. The observed surface temperature and the lapse rate structure of Venus' lower atmosphere can be matched quite closely with one-dimensional radiative-convective equilibrium models that incorporate the solar net flux profiles and gas and aerosol abundance data from the Pioneer Venus and Venera spacecraft missions. The more successful models of this type incorporate a water vapor mixing ratio of only several tens of ppm in the lowest regions of the atmosphere, in accord with the Venera spectrophotometer results. Hence it appears that the very high surface temperature of Venus is due almost entirely to the greenhouse effect.

5. The direct net thermal fiux measurements on the small probes indicate a radiative energy imbalance in the lower atmosphere when compared with net solar flux measurements. To the extent this comparison is significant, it suggests the downward transport of heat by processes in addition to solar radiation. A departure from radiative-convective equilibrium also may be indicated by the increase of static stability with decreasing altitude below about $18 \mathrm{~km}$ at low latitudes. The reality of this apparent imbalance is, however, questionable since the direct measurements of the net thermal fux from the three small probes differed widely, precluding a simple determination of the globally averaged net thermal flux deep in the atmosphere. In addition, questions remain about the thermal flux measurement errors in the lower atmosphere where the fluxes significantly exceed the most recent radiative transfer estimates.

6. High zonal wind velocities were directly measured in the four probes confirming the 4-day rotation of the atmosphere at the cloud levels needed to explain the low diurnal thermal contrasts. The drive for the winds is undoubtedly related to the observed high radiative flux divergences observed at cloud altitudes, but the exact mechanism is still unclear.

7. The role of both meridional and vertical dynamical heat transports in the thermal balance of Venus is not yet well defined, although new wind measurements are qualitatively in accord with requirements for maintaining relatively low equator to pole variations in temperature structure.

8. Radiative transfer calculations, based on measured temperatures and water vapor amounts, yield thermal flux profiles which differ enormously for water vapor profiles within the range of those found by PV gas chromatograph (LGC) and Venera 11 and 12. Models which reproduce the measured thermal net fluxes between 15 and $12 \mathrm{~km}$ require the presence of significant transmission windows in $\mathrm{CO}_{2}$ under the appropriate temperatures and pressures, and water vapor amounts somewhat less than that measured by Venera 11 
and 12. Improved knowledge of the opacity of $\mathrm{CO}_{2}$ and $\mathrm{H}_{2} \mathrm{O}$ under conditions of high temperature and pressure along with better information regarding the global water vapor distribution would lead to an improved understanding of the thermal budget of the lower atmosphere of Venus.

\section{REFERENCES}

Agee, E. M., T. S. Chen, and K. E. Dowell, A review of meso-scale cellular convection, Bull. Amer. Meteorol. Soc., 54, 1004-1012, 1973.

Avduevsky, V. S., M. Ya. Marov, B. E. Moshkin, and A. P. Ekonomov, Venera 8: Measurements of solar illumination through the atmosphere of Venus, J. Atmos. Sci., 30, 1215-1218, 1973.

Avduevsky, V. S., N. F. Borodin, V. P. Burtsev, Ya. V. Malkov, M. Ya. Marov, S. F. Morozov, M. K. Rozhdestvenskii, R. S. Romanov, S. S. Sokolov, V. G. Fokin, Z. P. Cheremukhina, and V. I. Shkirina, Automatic stations Venera 9 and Venera 10-Functioning of descent vehicles and measurement of atmospheric parameters, Cosmic Res., 14, 557-586, 1977.

Birnbaum, G., W. Ho, and A. Rosenberg, Far-infrared collision induced absorption in $\mathrm{CO}_{2}, \mathrm{II}$, Pressure dependence in the gas phase and absorption with liquid, J. Chem. Phys., 55, 1039-1045, 1971.

Boese, R. W., J. B. Pollack, and P. M. Silvaggio, First results from the large probe infrared radiometer experiment, Science, 203, 797-880, 1979.

Colin, L., Pioneer Venus program, J. Geophys. Res. this issue.

Counselman III, C. C., S. A. Gourevitch, R. W. King, G. B. Loriot, and R. G. Prinn, Venus winds are zonal and retrograde below the clouds, Science, 205, 85-87, 1979.

Counselman III, C. C., et al., Zonal and meridional circulation of the lower atmosphere of Venus determined by radio interferometry, $J$. Geophys. Res., this issue.

Gierasch, P. J., Meridional circulation and the maintenance of the Venus atmospheric rotation, J. Atmos. Sci., 32, 1038-1044, 1975.

Goody, R. M., and A. R. Robinson, A discussion of the deep circulation of the atmosphere of Venus, Astrophys. J., 146, 339-353, 1966.

Hansen, J. E., and J. W. Hovenier, Interpretation of the polarization of Venus, J. Atmos. Sci., 3I, 1137-1160, 1974.

Hoffman, J. H., V. I. Oyama, and U. von Zahn, Measurements of the Venus lower atmosphere composition: A critical comparison of results, J. Geophys. Res., this issue.

Holton, J. R., An Introduction to Dynamic Meteorology, Academic, New York, 1972.

Howard, H. T., et al., Venus: Mass gravity field, atmosphere, and ionosphere as measured by the Mariner 10 dual frequency radio system, Science, 183, 1297-1301, 1974.

Ingersoll, A. P., and J. B. Pechmann, Venus lower atmosphere heat balance, J. Geophys. Res., this issue.

Irvine, W. M., Monochromatic phase curves and albedos for Venus, $J$. Atmos. Sci., 25, 610-616, 1968.

Kálnay de Rivas, E., Further numerical calculations of the circulation of the atmosphere of Venus, J. Atmos. Sci., 32, 1017-1024, 1975.

Kawabata, K., D. L. Coffeen, J. E. Hansen, W. A. Lane, M. Sato, and L. D. Travis, Cloud and haze properties from Pioneer Venus polarimetry, $J$. Geophys. Res., this issue.

Keating, G. M., J. Y. Nicholson III, and L. R. Lake, Venus upper atmosphere structure, J. Geophys. Res., this issue.

Kliore, A. J., and I. R. Patel, The vertical structure of the atmosphere of Venus from Pioneer Venus radio occultations, J. Geophys. Res., this issue.

Knollenberg, R. G., and D. M. Hunten, Clouds of Venus: A preliminary assessment of microstructure, Science, 205, 70-74, 1979.

Knollenberg, R. G., and D. M. Hunten, The microphysics of the clouds of Venus: Results of the Pioneer Venus particle size spectrometer experiment, J. Geophys. Res., this issue.

Knollenberg, R. G., L. Travis, M. G. Tomasko, P. Smith, B. Ragent, L. Esposito, D. McCleese, J. Martonchik, and R. Beer, The clouds of Venus: A synthesis report, J. Geophys. Res., this issue.

Ksanfomaliti, L. W., Venera 9 and 10: Thermal radiometry, Icarus, $41,36-64,1980$.

Lane, W. A., Wavelength dependence of polarization, XXXV, Vertical structure of scattering layers above the visible Venus clouds, $A s-$ tron. J., 84, 683-691, 1979.

Marov, M. Ya., V. S. Avduevsky, V. V. Kerzhanovich, M. K. Rozhdestvensky, N. F. Borodin, and O. L. Ryabov, Venera 8: Measure- ments of temperature, pressure, and wind velocity on the illuminated side of Venus, J. Atmos. Sci., 30, 1210-1214, 1973.

Martonchik, J. V., and R. Beer, Analysis of spectrophotometric observations of Venus in the 3-4 micron region, J. Atmos. Sci, 32, 11511156, 1975.

Moroz, V. I., Yu. M. Golovin, A. P. Ekonomov, B. E. Moshkin, N. A. Parfent'ev, and N. F. Sanko, Spectrum of the Venus day sky, Nature, 284, 243, 1980.

Moshkin, B. E., A. P. Ekonomov, and Yu. M. Golovin, Spectral composition of the solar radiation in Venus' atmosphere according to light intensity measurement results on Venera 9 and Venera 10, Cosmic Res., 16, 412-418, 1978.

O'Leary, B., Venus: Vertical structure of stratospheric hazes from Mariner 10 pictures, J. Atmos. Sci., 32, 1091-1100, 1975.

Oyama, V. I., G. C. Carle, F. Woeller, J. B. Pollack, R. T. Reynolds, and R. A. Craig, Pioneer Venus gas chromatography of the lower atmosphere of Venus, J. Geophys. Res., this issue.

Palmer, K. F., and D. Williams, Optical constants of sulfuric acid: Application to the clouds of Venus?, Appl. Opt., 14, 208-219, 1975.

Pollack, J. B., A nongrey $\mathrm{CO}_{2}-\mathrm{H}_{2} \mathrm{O}$ greenhouse model of Venus, Icarus, 10, 314-341, 1969.

Pollack, J. B., and R. Young, Calculations of the radiative and dynamical state of the Venus atmosphere, J. Atmos. Sci., 32, 10251037, 1975.

Pollack, J. B., O. B. Toon, and R. Boese, Greenhouse models of Venus' high surface temperature, as constrained by Pioneer Venus measurements, $J$. Geophys. Res., this issue.

Ragent, B., and J. Blamont, The structure of the clouds of Venus: Results of the Pioneer Venus nephelometer experiment, $J$. Geophys. Res., this issue.

Rossow, W. B., A. D. Del Genio, S. S. Limaye, L. D. Travis, and P. H. Stone, Cloud morphology and motions from Pioneer Venus images, J. Geophys. Res., this issue.

Sagan, C., The radiation balance of Venus, Tech. Rep. N32-34, Jet Propulsion Lab., Pasadena, Calif., 1960.

Schubert, G., J. Apt, C. C. Counselmann III, C. Covey, A. Del Genio, L. S. Elson, G. Keating, A. J. Kliore, S. S. Limaye, H. E. Revercomb, A. Seiff, L. A. Sromovsky, V. E. Suomi, F. Taylor, R. Woo, R. E. Young, and U. von Zahn, Structure and circulation of the Venus atmosphere, J. Geophys. Res., this issue.

Seiff, A., D. B. Kirk, S. C. Sommer, R. E. Young, R. C. Blanchard, D. W. Juergens, J. E. Lepetich, P. F. Intrieri, J. T. Findlay, and J. S. Dein, Structure of the atmosphere of Venus up to 110 kilometers: Preliminary results from the four Pioneer Venus entry probes, Science, 203, 787-790, $1979 a$.

Seiff, A., D. B. Kirk, R. E. Young, S. C. Sommer, R. C. Blanchard, J. T. Findlay, and G. M. Kelly, Thermal contrast in the atmosphere of Venus: Initial appraisal from Pioneer Venus probe data, Science, 205, 46-49, $1979 b$.

Seiff, A., D. B. Kirk, R. E. Young, R. C. Blanchard, J. T. Findlay, G M. Kelly, and S. C. Sommer, Measurements of thermal structure and thermal contrasts in the atmosphere of Venus and related dynamical observations-Results from the four Pioneer Venus probes, J. Geophys. Res., this issue.

Shen, C.-H., and G. Springer, Photochemical particulate formation in sulfur dioxide-air mixtures, Atmos. Environ., 10, 235-239, 1976.

Stone, P. H., The dynamics of the atmosphere of Venus, J. Atmos. Sci., 32, 1005-1016, 1975.

Suomi, V. E., Cloud motions on Venus, The Atmosphere of Venus, NASA Spec. Publ., SP-382, 42-58, 1974.

Suomi, V. E., L. A. Sromovsky, and H. E. Revercomb, Preliminary results of the Pioneer Venus small probe net flux radiometer experiment, Science, 205, 82-85, 1979.

Suomi, V. E., L. A. Sromovsky, and H. E. Revercomb, Net radiation in the atmosphere of Venus: Measurements and interpretation, $J$. Geophys. Res., this issue.

Taylor, F. W., D. J. McCleese, and J. T. Diner, Polar clearing in the Venus clouds observed from the Pioneer orbiter, Nature, 279, 613614, $1979 a$.

Taylor, F. W., D. J. Diner, L. S. Elson, M. S. Hanner, D. J. McCleese, J. V. Martonchik, P. E. Reichley, J. T. Houghton, J. Delderfield, J. T. Schofield, S. E. Bradley, and A. P. Ingersoll, Infrared remote sounding of the middle atmosphere of Venus from the Pioneer orbiter, Science, 203, 779-781, 1979b.

Taylor, F. W., D. J. Diner, L. S. Elson, D. J. McCleese, J. J. Martonchik, J. Delderfield, S. P. Bradley, J. T. Schofield, J. C. Gille, and 
M. T. Coffey, Temperature, cloud structure, and dynamics of Venus middle atmosphere by infrared remote sensing from Pioneer orbiter, Science, 205, 65-67, 1979c.

Taylor, F. W., et al., Structure and meteorology of the middle atmosphere of Venus: Infrared remote sensing from the Pioneer orbiter, J. Geophys. Res., this issue.

Tomasko, M. G., R. Boese, A. P. Ingersoll, A. A. Lacis, S. S. Limaye, J. B. Pollack, A. Seiff, A. I. Stewart, V. E. Suomi, and F. W. Taylor, The thermal balance of the atmosphere of Venus, Space Sci. Rev., 20, 389-412, 1977.

Tomasko, M. G., L. R. Doose, and P. H. Smith, Absorption of sunlight in the atmosphere of Venus, Science, 205, 80-82, 1979.

Tomasko, M. G., L. R. Doose, Peter H. Smith, and A. P. Odell, Measurements of the flux of sunlight in the atmosphere of Venus, $J$. Geophys. Res., this issue.

Toon, O. B., R. P. Turco, and R. C. Whitten, A physico-chemical model of the Venus clouds, Bull. Am. Astron. Soc., 11, 544, 1979.

Travis, L. D., D. L. Coffeen, A. D. Del Genio, J. E. Hansen, K. Kawabata, A. A. Lacis, W. A. Lane, S. S. Limaye, W. B. Rossow, and P. H. Stone, Cloud images from Pioneer Venus orbiter, Science, 205, 74-76, 1979.

Vonder Haar, T. H., and V. E. Suomi, Measurements of the earth's radiation budget from satellites during a five year period, I, Extended time and space means, J. Atmos. Sci, 28, 305-314, 1971.

Whitby, K. T., The physical characteristics of sulfur aerosols, Atmos. Environ., 12, 135-159, 1978.

Young, L. G., Transmission of the atmosphere of Venus: The $\mathrm{CO}_{2}$ bands at 10 microns, Bull. Am. Astron. Soc., 11, 546, 1979.

Young, R. E., and J. B. Pollack, A three-dimensional model of dynamical processes in the Venus atmosphere, J. Atmos. Sci., 34, 1315-1351, 1977.

Young, R. E., and G. Schubert, Dynamical aspects of the Venus 4day circulation, Planet. Space Sci., 21, 1563-1580, 1973.

(Received February 4, 1980;

revised May 18, 1980;

accepted May 21, 1980.) 